\title{
Parapneumonic effusion and empyema
}

\author{
H. Hamm*, R.W. Light ${ }^{+}$
}

Parapneumonic effusion and empyema. H. Hamm, R.W. Light. @ERS Journals Ltd 1997. ABSTRACT: Parapneumonic effusions account for about one third of all pleural effusions. Approximately $40 \%$ of patients with pneumonia develop a concomitant effusion, which is associated with an increased morbidity and mortality.

In order to select the most appropriate therapy for the individual patient, the effusion should be categorized as being in the exudative, fibropurulent, or organizational stage, and all necessary information should be compiled to decide whether the effusion is likely to take an uncomplicated or a complicated course. There is a considerable variation in the aggressiveness and course of parapneumonic effusions, and, therefore, the spectrum of the appropriate therapy may vary from a conservative approach in uncomplicated effusions to aggressive surgical intervention in advanced multiloculated empyemas.

This review discusses current diagnostic and therapeutic options and offers guidelines for treating the various stages of parapneumonic effusions and empyemas. Eur Respir J 1997; 10: 1150-1156.
*Abt. Pneumologie, Medizinische Universitätsklinik, Freiburg, Germany. +University of California, Irvine, Pulmonary Exercise Laboratory, Veterans Administration Medical Center, Long Beach, CA, USA.

Correspondence: H. Hamm

Abt. Pneumologie

Medizinsiche Universitätsklinik

Hugstetter Str. 55

D-79106 Freiburg

Germany

Keywords: Empyema, pleural effusion, pleurisy, pleuritis, pneumonia

Received: December 191996

Accepted for publication January 231997

\section{Definitions}

"Pleurisy" (syn. pleuritis) is best defined as an inflammatory process of the pleura, which can be caused either by a variety of infectious microorganisms or by other inflammatory mechanisms. It is usually associated with localized chest pain that is synchronous with the respiratory cycle and is often manifested as a pleural rub on auscultation. It may induce an exudative pleural effusion. The pain and the rub sometimes subside when an effusion develops.

A "parapneumonic effusion" is an accumulation of exudative pleural fluid associated with an ipsilateral pulmonary infection.

"Uncomplicated parapneumonic effusions" are not infected and do not usually need tube thoracostomy.

"Complicated parapneumonic effusions" are usually associated with the pleural invasion of the infectious agent and require tube thoracostomy and sometimes decortication for their resolution.

An effusion is called an "empyema" when the concentration of leucocytes becomes macroscopically evident as a thick and turbid fluid (pus). In more than $50 \%$ of cases, it is of parapneumonic origin. Other common causes include surgical procedures (mainly thoracic surgery), traumas and oesophageal perforation.

\section{Pathophysiology}

Parapneumonic effusions and empyemas usually develop along the following lines.

\section{The pleuritis sicca stage}

The inflammatory process of the pulmonary parenchyma extends to the visceral pleura, causing a local pleuritic reaction. This leads to a pleural rub and the characteristic pleuritic chest pain, which originates from the sensitive innervation of the adjacent parietal pleura. A significant number of patients with pneumonia report pleuritic chest pain without developing a pleural effusion [1], suggesting that the involvement of the pleura may be limited to this stage in many cases of pneumonia.

\section{The exudative stage}

The ongoing inflammatory process leads to a mediator-induced increased permeability of local tissue and of regional capillaries. The subsequent accumulation of fluid in the pleural space is probably the combined result of the influx of pulmonary interstitial fluid [2] and of a local microvascular exudate. The fluid is usually clear and sterile, cytological specimens show a predominance of neutrophils, the $\mathrm{pH}$ is normal and the lactate dehydrogenate $(\mathrm{LDH})$ activity is $<1,000$ international units (IU).

\section{The fibropurulent stage}

This stage may develop quickly (within hours) in patients who are not receiving antibiotics, or who are

Previous articles in this series: No. 1: G. Miserocchi. Physiology and pathophysiology of pleural fluid turnover. Eur Respir J 1997; $10: 219-225$. No. 2: R.W. Light. Diagnostic principles in pleural disease. Eur Respir J 1997; 10: 476-481. No. 3: G.T. Kinasewitz. Transudative effusions. Eur Respir J 1997; 10: 714-718. No. 4: J. Ferrer. Pleural tuberculosis. Eur Respir J 1997; 10: $942-947$. 
treated with ineffective antibiotics. It is characterized by the deposition of fibrin clots and fibrin membranes ("sails") in the pleural space, which lead to loculations with increasing numbers of isolated collections of fluid. It is usually accompanied by (and caused by) bacterial invasion from the pulmonary parenchyma. The fluid is often turbid or frank pus. Cytology shows neutrophils and often degenerated cells, and Gram stains and bacterial cultures are usually positive. The metabolic and cytolytical activity in these effusions is high, as reflected by low $\mathrm{pH}$ values $(<7.2)$, and high $\mathrm{LDH}$ activities (often $>1,000 \mathrm{IU}$ )

\section{The organizational stage}

This final stage is characterized by the invasion of fibroblasts, leading to the transformation of interpleural fibrin membranes into a web of thick and nonelastic pleural peels. Functionally, gas exchange is often severely impaired on the side of the organizing empyema ("trapped lung"). The further course may vary from spontaneous healing with persistent defects of lung function to chronic forms of empyema with high risks for further complications, such as bronchopleural fistula, lung abscess, or "empyema necessitatis" (spontaneous perforation through the chest wall).

\section{Bacteriology}

All patients suspected of having a parapneumonic effusion should undergo a thoracentesis, unless the effusion is very small (fig. 1). Bacteriological studies should include a Gram stain and aerobic and anaerobic cultures. Many different bacteria have been identified as causative agents for parapneumonic effusions or empyemas. The spectrum of the most common organisms seems to have changed repeatedly over recent decades, influenced, in part, by the introduction of new antibiotics for the therapy of pneumonias [3]. Additionally, the reported spectra depend on the patient populations that have been studied by various investigators. According to recent series from the United States [4, 5] and Europe [6], the majority of culture-positive effusions are due to aerobic organisms, while up to $15 \%$ are caused exclusively by anaerobic bacteria, and the remainder are due to multiple, usually both aerobic and anaerobic, organisms. Streptococci (often Streptococcus pneumoniae) and Staphylococci (mostly Staphylococcus aureus) usually dominate aerobic Gram-positive isolates, whilst Escherichia coli, Klebsiella spp., Pseudomonas spp., and Haemophilus influenzae are the most common aerobic Gram-negative isolates. E. coli and anaerobic organisms are often found in combination with other organisms. The most frequent anaerobic isolates are Bacteroides spp. and Peptostreptococcus. Occasionally, Actinomyces spp., Nocardia spp., or fungi (most frequently Aspergillus) may be the cause of an empyema.

\section{Clinical aspects and differential diagnosis}

The clinical presentation of patients with pneumonia, whether or not they have parapneumonic effusions, is similar. In a large series by LIGHT and co-workers [1],

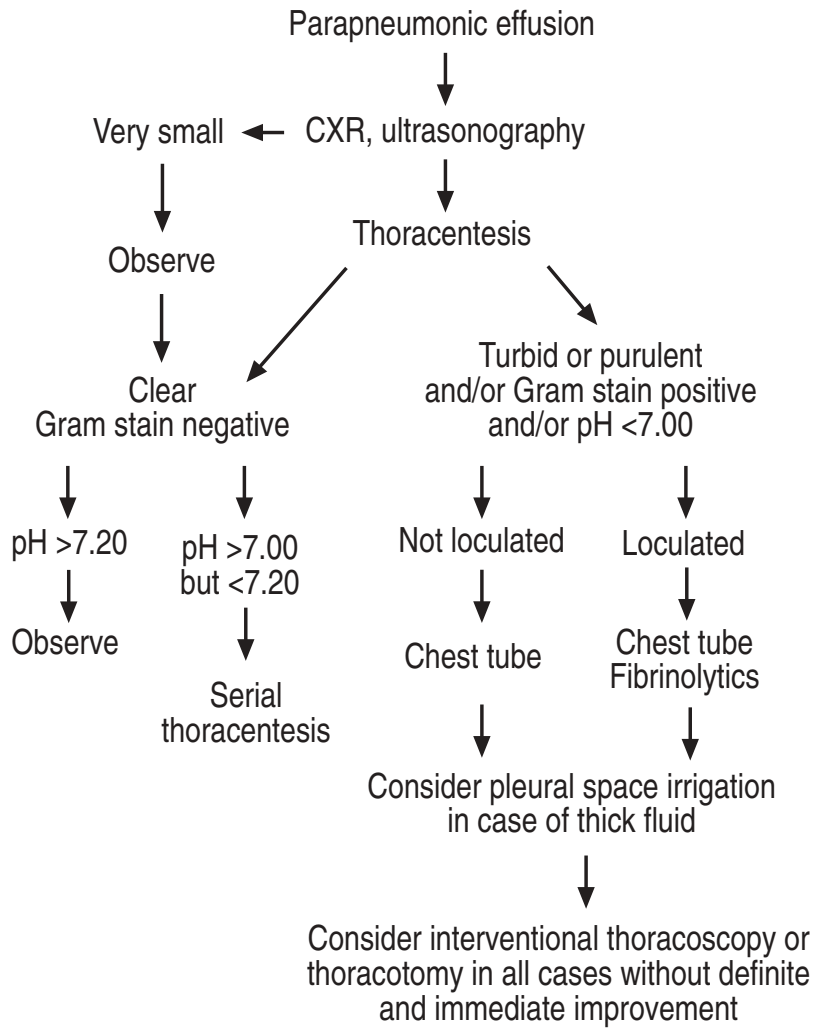

Fig. 1. - Approach to rational management of parapneumonic effusions and empyemas. CXR: chest radiography.

there were no significant differences between these two groups of patients regarding white blood cell count (WBC) and the occurrence of pleuritic chest pain.

Patients with pneumonia due to infection with aerobic bacteria usually suffer from an acute febrile illness, whilst patients with anaerobic infections tend to present with a more subacute or chronic condition, with a longer duration of symptoms and frequent weight loss [7]. Anaerobic pleuropulmonary infections often follow aspiration of oral or gastric contents. These patients usually have poor oral hygiene (foetor ex ore!) with anaerobic colonization of the oropharynx, and often suffer from conditions that predispose to aspiration, such as seizure disorders, syncopes of other origin, or alcoholism. The latter has been found to be a relevant associated disorder in as many as $29 \%$ [6] to $40 \%$ [5] of cases.

In general, patients who have a longer history of symptoms before seeking medical attention, or who have received insufficient treatment, are more likely to have complicated parapneumonic effusions or empyemas.

The finding of a purulent effusion without pneumonia may be explained as a postpneumonic empyema, in which the pulmonary infiltrates have already resolved. However, pleural empyemas are not necessarily caused by pneumonias (table 1). The majority of nonpneumonic empyemas are of iatrogenic origin, most commonly as a complication of a pneumonectomy or other thoracic surgical procedures. Thoracic surgery is responsible for about $20 \%$ of all thoracic empyemas [8-10]. Around $5 \%$ occur following a thoracic trauma and 5\% following an oesophageal perforation (often iatrogenic). Thoracentesis and spontaneous pneumothorax are each the 
Table 1. - Causes of empyema in 319 patients from three series [8-10]

\begin{tabular}{lcr}
\hline Cause & Number & $\%$ \\
\hline Pulmonary infection & 177 & 55 \\
Thoracic surgery & 66 & 21 \\
Trauma & 18 & 6 \\
Oesophageal perforation & 15 & 5 \\
Spontaneous pneumothorax & 7 & 2 \\
Thoracentesis & 6 & 2 \\
Subdiaphragmatic infection & 4 & 1 \\
Sepsis & 4 & 1 \\
Miscellaneous or unknown & 22 & 7 \\
\hline Total & 319 & 100 \\
\hline
\end{tabular}

(Adapted, with permission, from [3]).

cause of about $2 \%$ of cases. Rarely (in approximately $1 \%$ ), abdominal infections can be the origin of a thoracic empyema. Most originate from the subdiaphragmatic region and occur after surgical procedures, most commonly a cholecystectomy or a splenectomy [11].

\section{Differential diagnosis}

Fever, pulmonary infiltrates, and a pleural effusion are not invariably due to pneumonia or to a complication of some surgical procedure. A very important differential diagnosis, which should always be considered, is pulmonary infarction. Pulmonary embolism is a common disorder and paraembolic effusions occur in $25-50 \%$ of cases [12]. The effusions may become infected and will then require treatment identical to complicated parapneumonic effusions. Other disorders which should be considered include tuberculosis (see review No. 4 in this series), lupus erythematosus and other autoimmune disorders [13], acute pancreatitis and other diseases of the gastrointestinal (GI) tract [14], and drug-induced pleuropulmonary disease [15]. The turbid or milky aspect of empyemas may sometimes lead to the misconception of a chylothorax or pseudochylothorax (for details, see review No. 6 in this series, in this issue of the Journal).

\section{Imaging techniques}

Conventional chest radiographs usually suggest the presence of a parapneumonic effusion when there is a pulmonary infiltrate with evidence of ipsilateral pleural fluid. For the latter, a lateral chest radiograph is particularly useful to detect significant blunting of the posterior costophrenic angle. Bilateral decubitus chest radiographs may help to separate dense pulmonary infiltrates from free flowing pleural fluid. The most typical sign of an empyema is an encapsulated effusion in an atypical position [16].

Ultrasonography is a good method to guide a thoracentesis or place a chest tube. It is especially useful for small effusions and in other circumstances which require precise targeting, such as loculated effusions. The usefulness of ultrasonography to gain information on the nature of an effusion was investigated in a prospective study of 320 patients (224 with exudates and 96 with transudates) by YANG et al. [17]. Essentially, these authors found that ultrasound can supply the clinician with the following information: transudates are always anechoic, but exudates may also be anechoic. Effusions are usually exudates when they are septated or show a complex or homogeneously echogenic pattern. Dense echogenic patterns are most often associated with haemorrhagic effusions or empyemas.

Computed tomography (CT) may also be used for image-guided drainage. In a series of 86 effusions of various causes, contrast-enhanced CT showed parietal pleural thickening in all cases of empyema and in 56\% of parapneumonic effusions [18]. Parietal pleural thickening indicated an exudate with high specificity (96\%), but the sensitivity was low and it was not possible to differentiate inflammatory from malignant disease. Another study of 25 empyemas [16] showed a typical encapsulated and biconvex configuration in 20 cases. Further characteristics were thickening and increased contrast uptake of the parietal pleura and increased density of the adjacent subcostal tissue. However, it was not possible to correlate the CT findings with stages of clinical empyema.

Magnetic resonance imaging (MRI), especially sagittal T1-weighed images, allows a detailed analysis of the layers of the chest wall and their possible infiltration by inflammatory or malignant processes. Uncomplicated parapneumonic effusions do not seem to induce visible changes of the chest wall, while malignant effusions are frequently associated with alterations of the peripleural fat layer and the innermost intercostal muscles [19]. Whilst these findings are helpful in the differential diagnosis of benign and malignant effusions, it remains likely that complicated effusions and empyemas will show infiltration of the chest wall similar to malignant disease.

In summary, newer imaging techniques supply us with more detailed morphological information and can provide clues to the possible nature and cause of an undiagnosed effusion, but they do not obviate the need for a thoracentesis or other invasive diagnostic procedures.

\section{Pleural fluid chemical analysis}

Parapneumonic effusions are exudates. This is documented by measuring the pleural fluid protein level and LDH activity (and application of the criteria of LIGHT and co-workers [20]), and/or the cholesterol level [21] (see review on "Diagnostic principles in pleural disease", No. 2 in this series). Of course, exudates are not necessarily parapneumonic, since they may be caused by any inflammatory or malignant pleural process. The finding of a transudate excludes a parapneumonic effusion and strongly suggests one of the following underlying conditions: heart failure, liver cirrhosis, or hypoproteinaemia.

Several pleural fluid parameters have been utilized to assess the severity and to predict the future course of a parapneumonic effusion. Patients with complicated parapneumonic effusions tend to have a lower pleural fluid $\mathrm{pH}$ and glucose level and a higher LDH activity [1, 2226]. The glucose concentration correlates directly with the $\mathrm{pH}$ [26]. The cause for pleural fluid acidosis and low glucose levels is the local metabolic activity of inflammatory cells and bacteria [27]. The superiority of the $\mathrm{pH}$ over glucose or LDH measurements in parapneumonic 
effusions was recently confirmed in a meta-analysis of seven studies, using receiver operating characteristic (ROC) statistical techniques [28]. Therefore, it may suffice to measure the $\mathrm{pH}$ alone, but it must be emphasized that it is only valid when measured properly, which means: 1) collection and transport under strictly controlled anaerobic conditions; and 2) immediate measurement in a calibrated blood gas machine. It should also be kept in mind that the pleural fluid $\mathrm{pH}$ may not be useful in patients with systemic $\mathrm{pH}$ alterations (e.g. in systemic acidosis) [22] and in infections due to Proteus spp., which can induce a local metabolic alkalosis [29].

A properly measured pleural fluid $\mathrm{pH}$ is a useful indicator for tube thoracostomy. However, the $\mathrm{pH}$ should always be interpreted in concert with other information, e.g. with the clinical situation, the presence and number of loculations, and bacteriological findings. For instance, if the Gram stain of the pleural fluid is positive, tube thoracostomy is indicated regardless of the $\mathrm{pH}$.

Various recommendations have been made for the use of the $\mathrm{pH}$ in parapneumonic effusions: a pleural fluid $\mathrm{pH}<7.00$ [3] or $<7.10$ [30] has been proposed as the appropriate cut-off line for early tube drainage. These patients have a higher risk for developing multiloculated and empyematous effusions with conservative treatment alone. Conservative treatment and close observation (repeat thoracentesis) have been recommended within a $\mathrm{pH}$ range of 7.00-7.20 [3] or 7.10-7.30 [30]. Pleural fluids with pH levels between 7.2 [3] and 7.3 [30] usually take a benign course, and can be treated with systemic antibiotics alone. In the aforementioned meta-analysis by HeFFner et al. [28], the decision thresholds to identify complicated effusions ranged between $\mathrm{pH} 7.21$ and 7.29.

What conclusions should be drawn from the data that we have? 1) it makes sense to measure the pleural fluid $\mathrm{pH}$ in all parapneumonic effusions except for those that are frankly purulent or have a positive Gram stain (immediate indication for tube drainage, regardless of the $\mathrm{pH}$ ); 2) glucose measurements do not add relevant information and are not essential, unless there is doubt about the quality of the $\mathrm{pH}$ measurement; 3 ) $\mathrm{pH}$ values $<7.0$ should usually lead to tube drainage, in all other cases the $\mathrm{pH}$ should not be the sole criterion to decide on the necessity of a chest tube (see fig. 1); 4) effusions with $\mathrm{pH}$ 7.0-7.2 should be observed closely (repeat thoracocentesis); and 5) effusions with $\mathrm{pH}>7.2$ should be observed; those with $\mathrm{pH}$ values $>7.3$ are very unlikely to take a complicated course.

\section{Cytology}

Cytological specimens should be obtained in all cases of suspected parapneumonic effusions. True parapneumonic effusions and empyemas are invariably dominated by polymorphonuclear leucocytes. Any other findings strongly suggest another diagnosis (e.g. predominance of lymphocytes in an exudate is most often associated with tuberculosis or malignancy).

\section{Antibiotics and chest tube drainage}

The mainstay of all therapies in parapneumonic effusions and empyemas is systemic antibiotic therapy. It should be started as soon as pleural fluid specimens, sputum and blood samples, have been obtained for bacteriological studies. Empirical regimens should include antibiotics that are likely to be active against the bacteria that commonly cause parapneumonic effusions (see "Bacteriology"). The smell of the fluid (e.g. foetid odour in anaerobic infections) and other clinical circumstances (see "Clinical aspects and differential diagnosis") can influence the decision on the initial antibiotic regimen. In patients with community-acquired pneumonia, the regimen should consist of a second or third generation cephalosporin, or a beta-lactam/beta-lactamase inhibitor combination, and metronidazole or clindamycin should be added, if there is a possibility of an anaerobic infection. A macrolide, such as erythromycin or clarithromycin, may be part of the regimen in suspected Legionella infections, which may cause parapneumonic effusions, although they rarely tend to be complicated [3]. In patients with severe nosocomial pneumonia, third generation cephalosporins, or imipenem (which includes ac-tivity against anaerobic organisms) are reasonable first choices. In suspected Gram-negative infections, aminoglycosides may not be effective in the setting of a complicated purulent, acidotic and low partial pressure of oxygen $\left(\mathrm{P}_{2}\right)$ environment [30], and alternatives, such as aztreonam, should be considered in the initial combination. Further antibiotic treatment should immediately be adjusted to the results of the Gram stains and cultures.

A chest tube should be inserted in all complicated effusions, according to the criteria outlined above (see also fig. 1). It is best to place the tube under ultrasound (or CT) guidance to ensure its optimal localization. In loculated effusions, it may sometimes be advantageous to place a second tube into a separate fluid chamber. Traditionally, large tube sizes (26-32 French) have been recommended, but smaller tubes often prove sufficient, at least when the fluid does not consist of thick pus. In thick empyematous fluid, it may be helpful to place a double lumen catheter and irrigate the pleural space with saline solution [31] (fig. 1). At present, the additional application of local antibiotics via a chest tube is not recommended outside controlled trials [3].

The effectiveness of the chest tube drainage should be demonstrable within $24 \mathrm{~h}$ (reduction of radiological size, clinical improvement). Prolonged and unsuccessful tube drainage is associated with increased morbidity and mortality [32]; therefore, failure of this procedure should soon lead to a decision to institute a more aggressive approach.

\section{Fibrinolytic agents}

As early as 1949, TILlETt and SHERRY [33] recommended the use of fibrinolytics in loculated pleural effusions to induce enzymatic lysis of adhesions and debridement. It was proposed that such intrapleural therapy could obviate the need for surgical interventions. Today, fibrinolytic agents are recommended by many authors when chest tube drainage and a course of adequate antibiotics have failed to improve the situation, and when the effusion is loculated. A number of small uncontrolled studies have been published [34-43], which have described 
success rates ranging 60-95\%. The varying results of these studies are due, in part, to different patient characteristics and protocols, but also to various definitions of complete or partial resolution and treatment failure. In a larger recent series of 118 patients [44], fibrinolytic therapy was used in $83 \%$. The overall success rate (resolution without need for decortication) was reported to be $94 \%$. However, it is difficult to interpret some of these data, since the study included 23 cases of haemothorax, and the indications for drainage and intracavitary fibrinolytic therapy were variable. By contrast, in a retrospective comparison of various methods of treating empyema, only 2 out of 8 patients $(25 \%)$ treated with intracavitary fibrinolytics had a successful outcome [5].

Optimal dosage and timing of intrapleural fibrinolytic therapy are unknown. Most studies have used single doses of $250,000 \mathrm{IU}$ streptokinase or $100,000 \mathrm{IU}$ urokinase. Recently, Bouros et al. [43] reported successful therapy with single daily doses of 50,000 IU urokinase. This would help to reduce the costs, if the efficacy of this low dose can be confirmed. Usually, the procedure is carried out as follows: the fibrinolytic substance is diluted in $100 \mathrm{~mL}$ saline and instilled via the chest tube. The tube is then clamped for $1-4 \mathrm{~h}$. The instillation is usually repeated once daily, and is continued for several days, sometimes for periods of up to 2 weeks. Many authors report that the therapy enhances the drainage volume [38, 40, 41, 43], which is generally attributed to the effectiveness of fibrinolysis. However, a recently published animal study [45] suggested that streptokinase induces the production of additional pleural fluid. Furthermore, this study confirmed that streptokinase reduces the number of interpleural adhesions, but it failed to demonstrate a significant reduction of plaques on the pleural surfaces.

Intrapleural fibrinolytic treatment does not seem to have a measurable influence on systemic coagulation parameters (e.g. [46]). However, Temes et al. [42] in their series observed a case of significant local bleeding which required thoracotomy. There may be a risk of allergic reactions after repeated use of streptokinase; however, clinically relevant side-effects seem to be rare. Thus, intrapleural fibrinolytic treatment may be considered safe. It has also been successfully used in children [38].

In summary, presently available evidence suggests that there is a place for local fibrinolytic therapy in complicated, loculated parapneumonic effusions or empyemas (fig. 1). It is probably important to start the treatment as early as possible, as soon as it becomes evident that a chest tube and antibiotics alone do not suffice in the control of the situation. At the same time, however, alternative options, such as interventional thoracoscopy or thoracotomy and decortication, have to be considered. Compared to these more invasive procedures, fibrinolytics certainly have the advantage of avoiding the risks of general anaesthesia, which may be an important aspect in the treatment of elderly and/ or multimorbid patients. The lack of controlled trials still leaves us with uncertainties as to when, for how long, and what dose of which fibrinolytic agent should be given. As long as these questions remain unresolved, intrapleural fibrinolytic therapy will continue to be a matter of debate.

\section{Therapeutic thoracoscopy}

If a complicated parapneumonic effusion or an empyema does not respond to chest tube drainage, antibiotics, and, possibly, thrombolytic agents, thoracoscopic lysis of adhesions and debridement can be the next step. Whilst it is considered to be a minimal invasive procedure, it usually requires general anaesthesia. Like fibrinolytic therapy, thoracoscopy seems to be most successful when carried out early in the disease [47]. It must be kept in mind that debridement may not be easy through the thoracoscope, and it may fail in cases of extensive pleural adhesions [48]. Therefore, thoracoscopic procedures must sometimes be extended to formal thoracotomy. Successful therapeutic thoracoscopies have also been reported in children $[49,50]$.

\section{Thoracotomy with decortication}

Prolonged unsuccessful tube drainage of pleural empyemas is associated with increased morbidity and mortality. Therefore, some authors recommend an early aggressive surgical approach after a short trial of tube drainage and antibiotics [32]. It has a high success rate (up to 95\%) [5], whilst morbidity and mortality are low. However, formal thoracotomy remains a major thoracic operation, with some intra- and peri-operative risks, which become particularly relevant in patients who are markedly debilitated. Thus, the decision to initiate this procedure should only be made after considering the prospects of fibrinolytic therapy or therapeutic thoracoscopy, if available (fig. 1). Certainly, chronic postpneumonic empyemas are a major indication for formal thoracotomy [51]. They are often characterized by delayed presentation, delayed treatment, and complicating underlying conditions, such as chronic alcoholism. Advanced pleural fibrosis is very likely under these circumstances, thus reducing the chances for alternative procedures, such as fibrinolysis or thoracoscopic debridement. Thoracotomy and decortication are very effective procedures in such cases [51]. Generally, it should be kept in mind that control of the infection, but not the impairment of lung function, is the only imperative reason for decortication in the first weeks of treating an empyema. With the control of the infection by other means, even thick pleural peels can resolve gradually, and lung function parameters can return to normal after several months. Thus, late decortication to repair persisting defects of lung function is usually only performed after several months of monitoring the further course of such patients.

\section{Open drainage}

Open drainage is a procedure which is sometimes carried out in patients whose risk appears too high to tolerate thoracoscopy or formal thoracotomy. Thus, it is most often performed in elderly, polymorbid patients with advanced, multiloculated empyemas. It is a lengthy procedure, with treatment periods lasting up to several months. Therefore, intrapleural fibrinolytic therapy should be attempted before a final decision for open drainage is made. Moreover, it should be ensured that 
there is sufficient fusion between the lung and the chest wall. Usually, a prolonged inflammatory process in the pleural space will lead to satisfactory coupling of these two structures. This is important, to avoid the risk of a pneumothorax when opening the pleural cavity permanently for free communication with the atmosphere. This risk should be tested for by opening the closed chest tube to the atmosphere and looking for a pneumothorax on a chest radiograph.

\section{Summary}

The presence of a parapneumonic effusion increases the morbidity and mortality of patients with pneumonia. In order to select the most appropriate therapy for the individual patient, the effusion should be categorized as being in the exudative, fibropurulent or organizational stage and, from a more clinical point of view, all necessary information should be compiled to decide whether the effusion is likely to take an uncomplicated or a complicated course. This decision is based upon biochemical and bacteriological pleural fluid analyses, and on ultrasound and/or CT scan imaging of the pleural cavity.

The best way to treat an uncomplicated parapneumonic effusion is early and appropriate antibiotic therapy, which often prevents a complicated or empyematous course.

A complicated effusion or an empyema requires immediate chest tube drainage. Substantial clinical improvement and resolution of the effusion should be documentable within the next $24 \mathrm{~h}$. Otherwise, it is likely that a fibropurulent or organizational stage has been reached and that loculations have developed. At this time, three further treatment modalities need to be considered: 1) Intra-pleural fibrinolytic therapy; 2) thoracoscopic debridement; or 3 ) early thoracotomy and decortication.

Fibrinolytic therapy may obviate the need for surgical intervention in many cases. However, we are still lacking controlled trials to support this view more clearly. Usually, fibrinolytic therapy should be declared unsuccessful when the effusion has not completely cleared within 1 week. Thoracoscopic debridement is usually considered the next step in centres that are equipped for this procedure. However, it should always be performed with the option of switching to thoracotomy, since technical difficulties in removing all adhesions occur in a substantial number of cases, with success rates of only around $60 \%$. Formal thoracotomy with decortication is the most radical approach and remains the gold standard in the treatment of complicated parapneumonic effusions and empyemas, with success rates well above $90 \%$. It is an attractive option for early and decisive treatment in patients who are good surgical candidates, and it remains the initial treatment of choice in all chronic, multiloculated empyemas.

Open drainage is a lengthy and comparably unattractive procedure, which is reserved for some patients who are too debilitated to tolerate a more invasive approach.

It should be kept in mind that the primary goal of all initial medical or surgical procedures is the control of the pleural infection. Considerations based on imaging techniques or lung function results are only of secondary importance at this time. Therefore, pleural adhesions and restricted lung function alone may not justify immediate invasive procedures, if pleural infection is being controlled. In such cases, the further course should be observed, because spontaneous restitutio ad integrum is probable. Late decortication may be indicated in cases of persisting and significant loss of lung function.

\section{References}

1. Light RW, Girard WM, Jenkinson SG, George RB. Parapneumonic effusions. Am J Med 1980; 69: 507-511.

2. Wiener-Kronish JP, Sakuma T, Kudoh I, et al. Alveolar epithelial injury and pleural empyema in acute $P$. aeruginosa pneumonia in anesthetized rabbits. J Appl Physiol 1993; 75: 1661-1669.

3. Light RW. Pleural diseases. 3rd edn. Baltimore, Williams \& Wilkins, 1995.

4. Brook I, Frazier EH. Aerobic and anaerobic microbiology of empyema: a retrospective review in two military hospitals. Chest 1993; 103: 1502-1507.

5. LeMense GP, Strange C, Sahn SA. Empyema thoracis: therapeutic management and outcome. Chest 1995; 107: 1532-1537.

6. Alfagame I, Munoz F, Pena N, Umbria S. Empyema of the thorax in adults: etiology, microbiologic findings, and management. Chest 1993; 103: 839-843.

7. Bartlett JG, Finegold SM. Anaerobic infections of the lung and pleural space. Am Rev Respir Dis 1974; 110: 56-77.

8. Yeh TJ, Hall DP, Ellison RG. Empyema thoracis: a review of 110 cases. Am Rev Respir Dis 1963; 88: 785-790.

9. Snider GL, Saleh SS. Empyema of the thorax in adults: review of 105 cases. Chest 1968; 54: 12-17.

10. Smith JA, Mullerworth MH, Westlake GW, Tatoulis J. Empyema thoracis: 14 year experience in a teaching center. Ann Thorac Surg 1991; 51: 39-42.

11. Sahn SA. The pleura. Am Rev Respir Dis 1988; 138: 184-234.

12. Heffner JE. Pleural effusions from pulmonary thromboembolism. Semin Respir Med 1987; 9: 59-64.

13. Joseph J, Sahn SA. Connective tissue disease and the pleura. Chest 1993; 104: 262-270.

14. Light RW. Exudative pleural effusions secondary to gastrointestinal diseases. Clin Chest Med 1985; 6: 103-108.

15. Rosenow EC. Drug-induced bronchopulmonary pleural disease. J Allergy Clin Immunol 1987; 80: 780-787.

16. Kirsch E, Guckel C, Kaim A, Steinbrich W. The findings and value of computed tomography in pleural empyema. Rofo Fortschr Geb Röntgenstr Neuen Bildgeb Verfahr 1994; 161: 404-411.

17. Yang PC, Luh KT, Chang DB, Wu HD, Yu CJ, Kuo $\mathrm{SH}$. Value of sonography in determining the nature of pleural effusions: analysis of 320 cases. Am J Roentgenol 1992; 159: 29-33.

18. Aquino SL, Webb WR, Gushiken BJ. Pleural exudates and transudates: diagnosis with contrast-enhanced CT. Radiology 1994; 192: 803-808.

19. Bittner RC, Schnoy N, Schönfeld N, et al. High-resolution magnetic resonance tomography (HR-MRT) of the pleura and thoracic wall: normal findings and pathological findings. Rofo Fortschr Geb Röntgenstr Neuen Bildgeb Verfahr 1995; 162: 296-303.

20. Light RW, MacGregor MI, Luchsinger PC, Ball WC. 
Pleural effusions: the diagnostic separation of transudates and exudates. Ann Intern Med 1972; 77: 507-513.

21. Hamm H, Bröhan U, Böhmer R, Missmahl HP. Cholesterol in pleural effusions: a diagnostic aid. Chest 1987; 92: 296-302.

22. Light RW, MacGregor M, Ball WC Jr, Luchsinger PC. Diagnostic significance of pleural fluid $\mathrm{pH}$ and $\mathrm{PCO}_{2}$. Chest 1973; 64: 591-596.

23. Potts DE, Levin DC, Sahn SA. Pleural fluid $\mathrm{pH}$ in parapneumonic effusions. Chest 1976; 70: 328-331.

24. Poe RH, Marin MG, Israel RH, Kallay MC. Utility of pleural fluid analysis in predicting tube thoracostomy/ decortication in parapneumonic effusions. Chest 1991; 100: 963-967.

25. Good JT, Taryle DA, Maulitz RM, Kaplan RL, Sahn $\mathrm{SA}$. The diagnostic value of pleural fluid $\mathrm{pH}$. Chest 1980; 78: 55-59.

26. Potts DE, Levin DC, Sahn SA. The glucose-pH relationship in parapneumonic effusions. Arch Intern Med 1978; 138: 1378-1380.

27. Sahn SA, Reller LB, Taryle DA, Antony VB, Good JT Jr. The contribution of leucocytes and bacteria to the low pH of empyema fluid. Am Rev Respir Dis 1983; 128: 811-815.

28. Heffner JE, Brown LK, Barbieri C, DeLeo JM. Pleural fluid chemical analysis in parapneumonic effusions. Am J Respir Crit Care Med 1995; 151: 1700-1708.

29. Pine JR, Hollmann JL. Elevated pleural fluid $\mathrm{pH}$ in Proteus mirabilis empyema. Chest 1983; 84: 109-111.

30. Sahn SA. Management of complicated parapneumonic effusions. Am Rev Respir Dis 1993; 148: 813-817.

31. Rosenfeldt FL, McGibney D, Braimbridge MV, Watson DA. Comparison between irrigation and conventional treatment for empyema and pneumonectomy space infection. Thorax 1981; 36: 272-277.

32. Pothula V, Krellenstein DJ. Early aggressive surgical management of parapneumonic empyemas. Chest 1994; 105: 832-836.

33. Tillett WS, Sherry S. Effect in patients of streptococcal fibrinolysin (streptokinase) and streptococcal deoxyribonuclease on fibrinous, purulent, and sanguineous exudations. J Clin Invest 1949; 28: 173-186.

34. Bergh NP, Ekroth R, Larsson S, Nagy P. Intrapleural streptokinase in the treatment of haemothorax empyema. Scand J Thorac Cardiovasc Surg 1977; 11: 265-268.

35. Moulton JS, Moore PT, Mencini RA. Treatment of loculated pleural effusions with transcatheter intracavitary urokinase. Am J Roentgenol 1989; 153: 941-945.

36. Lee KS, Im JG, Kim YH, Hwang SH, Bae WK, Lee $\mathrm{BH}$. Treatment of thoracic multiloculated empyemas with intracavitary urokinase: a prospective study. Radiology 1991; 179: 771-775.

37. Aye RW, Froese DP, Hill LD. Use of purified streptokinase in empyema and hemothorax. Am J Surg 1991; 161: 560-562.

38. Rosen H, Nadkarni V, Padman R, Klein J. Intrapleural streptokinase as adjunctive treatment for persistent empyema in pediatric patients. Chest 1993; 103: 1190-1193.

39. Robinson LA, Moulton AL, Fleming WH, Alonso A, Galbraith TA. Intrapleural fibrinolytic treatment of multiloculated thoracic empyemas. Ann Thorac Surg 1994; 57: 803-814.

40. Pollak JS, Passik CS. Intrapleural urokinase in the treatment of loculated pleural effusions. Chest 1994; 105: 868-873.

41. Taylor RF, Rubens MB, Pearson MC, Barnes NC. Intrapleural streptokinase in the management of empyema. Thorax 1994; 49: 856-859.

42. Temes RT, Follis F, Kessler RM, Pett SB, Wernly JA. Intrapleural fibrinolytics in management of empyema thoracis. Chest 1996; 110: 102-106.

43. Bouros D, Schiza S, Tzanakis N, Drositis J, Siafakas N. Intrapleural urokinase in the treatment of complicated parapneumonic pleural effusions and empyema. Eur Respir J 1996; 9: 1656-1659.

44. Moulton JS, Benkert RE, Weisiger KH, Chambers JA. Treatment of complicated pleural fluid collections with image-guided drainage and intracavitary urokinase. Chest 1995; 108: 1252-1259.

45. Strange C, Allen ML, Harley R, Lazarchick J, Sahn SA. Intrapleural streptokinase in experimental empyema. Am Rev Respir Dis 1993; 147: 962-966.

46. Berglin E, Ekroth R, Teger-Nilsson AL, William-Olsson G. Intrapleural instillation of streptokinase: effects on systemic fibrinolysis. Thorac Cardiovasc Surg 1981; 29: 124-126.

47. Sendt W, Forster E, Hau T. Early thoracoscopic debridement and drainage as definitive treatment for pleural empyema. Eur J Surg 1995; 161: 73-76.

48. Ridley PD, Braimbridge MV. Thoracoscopic debridement and pleural irrigation in the management of empyema thoracis. Ann Thorac Surg 1991; 51: 461-464.

49. Kern JA, Rodgers BM. Thoracoscopy in the management of empyema in children. J Pediatr Surg 1993; 28: $1128-1132$.

50. Stovroff M, Teague G, Heiss KF, Parker P, Ricketts RR. Thoracoscopy in the management of pediatric empyema. J Pediatr Surg 1995; 30: 1211-1215.

51. Martella AT, Santos GH. Decortication for chronic postpneumonic empyema. J Am Coll Surg 1995; 180: 573-576. 